\title{
Cognitive and psychological profiles in treatment compliance: a study in an elderly population with hemophilia
}

\author{
Silvia Riva' \\ Alessandro Nobili² \\ Codjo D Djade ${ }^{2}$ \\ Maria Elisa Mancuso ${ }^{3}$ \\ Elena Santagostino ${ }^{3}$ \\ Gabriella Pravettoni ${ }^{1-4}$ \\ 'Department of Health Sciences, \\ Università degli Studi di Milano, Milan, \\ Italy; ${ }^{2}$ Department of Neuroscience, \\ IRCCS, Istituto di Ricerche \\ Farmacologiche "Mario Negri", \\ Milan, Italy; ${ }^{3}$ Angelo Bianchi Bonomi \\ Hemophilia and Thrombosis Centre, \\ IRCCS Ca' Granda, Policlinic Hospital, \\ Milan, Italy; ${ }^{4}$ European Institute of \\ Oncology, Milan, Italy
}

This article was published in the following Dove Press journal:

Clinical Interventions in Aging

9 July 2015

Number of times this article has been viewed

\begin{abstract}
Elderly patients with hemophilia have to face new challenges linked to concomitant pathologies and concurrent use of different treatments. In order to promote optimal care in the elderly hemophilia population, this study is aimed to analyze treatment compliance in relation to the presence of comorbidities and the role of potential determinants that can affect compliance (positively or negatively), including health-related quality of life, cognitive decline, and sociodemographic parameters (eg, living situation, partnership, presence of caregivers). This will be an observational study of elderly patients with hemophilia (aged $>60$ years). Patients will be interviewed during their routine medical visits. The data interview will pertaining to several dimension of treatment management. This study will detect more vulnerable patients with special care needs and will highlight psychological factors that should be considered for future psychosocial interventions.
\end{abstract}

Keywords: hemophilia, cognitive assessment, cognitive decline, compliance, treatment, aging

\section{Background}

For patients with hemophilia, life expectancy is now approaching that of the general male population, especially in regions and countries that have the resources for regular replacement therapy with coagulation-factor concentrates. ${ }^{1}$ In Italy, for example, nearly $8 \%$ of patients with severe hemophilia A or B are aged 65 years or older. ${ }^{2}$ The introduction of virus-inactivated plasma-derived coagulation factors and then of recombinant products has radically changed the treatment of patients with hemophilia, improving their health-related quality of life (HRQL) and life expectancy. ${ }^{3-6}$ As a consequence, a growing number of patients with hemophilia have reached an older age, and nowadays physicians in hemophilia centers "find that they must handle age-related clinical problems never previously observed in this population". ${ }^{7-8}$ The new issues for hemophilia care are thus to offer best possible health care for this elderly cohort of patients, who frequently present not only with the comorbidities usually linked with hemophilia (such as arthropathy, chronic pain, blood-borne infections), but also with general age-related conditions such as diabetes, cancer, or cardiovascular disease. ${ }^{8-9}$

Compliance with a medication regimen is defined as the extent to which patients take medications as prescribed by their health providers. ${ }^{3}$ Compliance is a complex behavioral construct highly influenced by the context and the experiences of the patients and by the care activities of their health providers. Noncompliance represents the extent to which the patient's behavior does not follow medical instructions or 
treatment rules. ${ }^{3-4,10}$ Compliance with treatment is, therefore, a crucial determinant of optimal health and quality of life. However, it has been reported that adult patients with chronic illness take only $50 \%$ of their prescribed medication. ${ }^{11-13}$ The situation in elderly patients seems even more problematic. Around the $90 \%$ of elderly patients make some medication errors, and $35 \%$ make potentially serious errors. ${ }^{14}$ Some of the explanations as to why elderly patients may experience difficulties taking their treatment properly and on time are connected to physical limitations (eg, fatigue, arthritis) and often to functional limitations, such as memory loss (they simply forget to take their medication on time), confusion (which occurs especially with multiple drugs and complex regimens), emotional problems such as anxiety or depression, low-quality HRQL, isolation, and scarce social support from caregivers.

Scarce compliance is also observed in persons with hemophilia and may affect treatment choice: a cross-sectional survey conducted in the United States reported that one-third of hemophilia physicians do not prescribe prophylaxis due to concerns about patient compliance with treatment. ${ }^{15}$ Based on these considerations, the scenario of compliance with treatment for elderly patients with hemophilia is emerging as deeply worrisome and needs to be evaluated.

\section{Methods}

\section{Primary and secondary objectives}

The primary objective of this study is to measure the level of compliance with treatment prescription (replacement therapy and multiple drugs for the treatment of multimorbidity) using the following instruments:

- The Hemophilia Satisfaction Questionnaire (Hemo-Sat for adults $)^{16}$

- The Self-Efficacy for Appropriate Medication Use Scale $^{17}$

- REPOSI Appropriateness Drug Prescription Interview. ${ }^{18}$ The secondary objectives of this study are to assess the determinants that affect compliance, including:

- The presence of cognitive dysfunction, assessed with the Mini-Mental State Examination ${ }^{19}$

- Clinical parameters (eg, bleeding frequency, severity of the disease, target joints [that is, three or more bleeds in the same joint during the previous 6 months])

- Sociodemographic parameters (eg, marital status, living situation, education, a series of ad hoc questions pertaining to daily activities, such as "How does the patient fill their day", "How do they go out", etc)

- The presence of caregivers
- HRQL, assessed with the Euro-QoL questionnaire $(\mathrm{EQ}-5 \mathrm{D}-\mathrm{L}){ }^{20}$

\section{Outcomes}

- The primary outcome is to estimate the number of vulnerable elderly patients at risk for poor treatment compliance because of cognitive decline, poor HRQL, or sociodemographic parameters (level of education, living situation, partnership, etc).

- The secondary outcome is identification of the main psychological factors that can be crucial to consider in planning future interventions aimed at supporting treatment management in elderly patients with hemophilia.

\section{Study design}

This is a prospective noninterventional study (NIS) that describes compliance in elderly patients who suffer from moderate or severe hemophilia (A or B) and who are receiving either on-demand treatment or prophylaxis. The study is focused on adults ( $>60$ years of age) living in Italy.

This NIS will be conducted and coordinated at the Hemophilia Centre of Milan (Italy). However, other Italian hemophilia and thrombosis centers will be asked to participate in this data collection under the supervision of the Hemophilia Centre of Milan. It is anticipated that data from approximately 80 patients will be collected.

\section{Patients}

The data of patients with a diagnosis of moderate or severe hemophilia (A or B), identified by the Hemophilia Centre of Milan, will be chosen for collection within this NIS. Patients should present the following characteristics:

- Male, with hemophilia A or B

- Severe or moderate factor deficiency ( $5 \%$ or less)

- Age $\geq 60$ years

- Able to read and understand study materials (patient information and data protection form, patient-related questionnaires)

- Having signed the informed consent and data protection form

- Able to write independently and to complete questionnaires.

\section{Withdrawal and replacement of patients}

Patients may withdraw from participation in this NIS at any time without the need to provide any explanation. Collection of patient data will be performed as long as source data are available and the patient's permission to document the 
data has not been withdrawn. No replacement for withdrawn patients will be performed.

\section{Design and timeframe}

In this prospective study, compliance will be assessed over a 12-month period in a cohort of adults with hemophilia who are receiving routine medical care. Compliance scores will be correlated with the results of routine hemophilia clinical assessments (eg, therapy for concomitant diseases, therapy for bleeding frequency, severity of hemophilia) and the results of sociodemographic data related to personal life circumstances (eg, perceived HRQL, number and type of caregivers).

\section{Variables assessed}

Different types of variables will be assessed in the current study, including compliance, HRQL, treatment satisfaction, and sociodemographic and clinical data. All the questions (self-administered) will be presented in a patient booklet for each individual patient. The questionnaire administration will last around 20 minutes per patient.

\section{Compliance}

The Hemo-Sat ${ }_{\mathrm{A}}$ Questionnaire represents the first hemophilia-specific treatment satisfaction questionnaire for adults developed in Italy. Treatment satisfaction has recently become a focus of interest, representing the individual rating of the process and outcomes of patients' treatment experience, and is related to adherence and willingness to continue treatment. The Hemo-Sat ${ }_{\mathrm{A}}$ consists of 34 items pertaining to six dimensions ("ease and convenience", “efficacy", "burden", “specialist”, “center", and "general satisfaction"). ${ }^{18}$

The Self-Efficacy for Appropriate Medication Use Scale was developed on the basis of Bandura's social cognitive theory. This instrument was developed for patients with a chronic disease. ${ }^{17}$ Patients are asked about how confident they were that they did take their medications accurately (unconfident $=1$, fairly confident $=2$, and extremely confident $=3$ ) . The lowest possible score of the 13-item questionnaire is 13 , and the highest possible score is 39 ; the high score indicates that the participant is highly confident about taking medication.

The REPOSI Appropriateness Drug Prescription Interview is a short new instrument that assesses the appropriateness of drug prescriptions in relation to polypharmacy..$^{18}$ It has recently been proposed and implemented in the context of the REPOSI (REgistro POliterapie, SIMI) Study, a noninterventional, multicenter registry designed to collect a set of clinical and laboratory data on elderly patients consecutively hospitalized. The interview is composed of nine questions that aim to review the number of drugs prescribed and to check their appropriateness.

\section{Cognitive profile}

The Mini-Mental State examination, or Folstein test, is a brief 30-point questionnaire that is used to screen for cognitive impairment. It is commonly used in medicine to screen for dementia. It is also used to estimate the severity of cognitive impairment and to follow the course of cognitive changes in an individual over time, thus making it an effective way to document an individual's response to treatment. ${ }^{19}$

\section{Health-related quality of life}

The EQ-5D-3L is a standardized instrument for use as a measure of health outcomes. Applicable to a wide range of health conditions and treatments, the EQ-5D health questionnaire provides a simple descriptive profile and a single index value for health status. The EQ-5D-3L consists of two pages, the EQ-5D descriptive system and a visual analog scale. The EQ-5D-3L descriptive system comprises the following five dimensions: mobility, self-care, usual activities, pain/discomfort, and anxiety/depression. Each dimension has three levels: no problems, some problems, extreme problems. ${ }^{20}$

Table 1 shows the clinical and sociodemographic data that will be obtained by the physician and inserted in the Medical Documentation Form.

Table I Clinical and sociodemographic data

\begin{tabular}{ll}
\hline Clinical data & Sociodemographic data \\
\hline Inclusion/exclusion criteria & $\begin{array}{l}\text { Marital status (past, current) } \\
\text { Partnership } \\
\text { Lemographics }\end{array}$ \\
Bleeding history & $\begin{array}{l}\text { geographical location) } \\
\text { Education (school level will be } \\
\text { Target joints }\end{array}$ \\
& $\begin{array}{l}\text { asked according to the International } \\
\text { Standard Classification of Education*) }\end{array}$ \\
Inhibitor history & $\begin{array}{l}\text { Professional situation } \\
\text { (eg, employment status) }\end{array}$ \\
Viral infections & Daily life activities functioning \\
Concomitant diseases & \\
Surgery & \\
Replacement therapy regimen & \\
Concomitant drugs other & \\
than replacement therapy & \\
Medical visits & \\
\hline
\end{tabular}

Note: *UNESCO developed the International Standard Classification of Education (ISCED) to facilitate comparisons of education statistics and indicators across countries on the basis of uniform and internationally agreed definitions. 


\section{Statistical analysis Sample size determination}

This is an NIS, and the statistical approach is exploratory in nature. A sample size of 80 patients aged $>60$ years has been estimated, for the following reasons:

1. The mean \pm standard deviation of the Hemo-Sat ${ }_{\mathrm{A}}$ in 29 adult patients with hemophilia (the only available measure on compliance in a cohort of hemophiliac patients) was reported as $21.4 \pm 25.7$. Scores range from 0 to 100 ; a lower score indicates higher satisfaction. ${ }^{21}$ Assuming that this variability is also representative of other groups, and further, that a relevant effect to be detected between ages (younger and older) amounts to 23 points on this scale, leading to an effect size of 0.75 , then $70-90$ patients should be enrolled per region (approximately 80 patients overall) with a precision of $95 \%$ (ie, $95 \%$ confidence interval [CI]). The power to detect significant differences between determinants (eg, cognitive decline) with a fairly balanced distribution in this case reaches $81 \%-96 \%$.

2. This seems to be a reasonable number to collect, taking into account that hemophilia is a rare disease and the prevalence of elderly hemophiliacs, even if increasing, is still limited in the Italian centers.

3. In this area, as cited by the review of Schrijvers et al ${ }^{22}$ on compliance in elderly hemophiliacs, the mean sample size of the five studies cited was 80 participants (range, 38-147 participants).

4. Statistically, this is a correlational study aimed to identify correlations and/or associations between aging and compliance. In a correlational study, a limited sample size is commonly accepted. ${ }^{23}$

\section{Statistical data}

The study objective is addressed in an exploratory manner to identify compliance in elderly patients with hemophilia and the determinants that strongly impact compliance (HRQL, cognitive assessment, the presence of caregivers, clinical parameters, sociodemographic parameters).

Compliance and determinants (HRQL, cognitive assessment, the presence of caregivers, clinical parameters, sociodemographic parameters) will be analyzed descriptively for each measure point by means of frequency, median, mean, standard deviation, range, and 95\% CIs. Compliance differences between different patient groups (eg, prophylaxis versus on-demand, polytherapy versus non-polytherapy, family status, personal life circumstances, etc.) will be assessed using inference statistical analysis (Mann-Whitney $U$-test or Kruskall-Wallis test for categorical determinants;
$P$-values $<0.05$ will be regarded as nominally significant in double-sided tests). For ordinal or continuous determinants, correlations and regressions will be calculated (Spearman or Pearson correlation) to identify a potential relationship between these factors (eg, number of bleeding events, sports activity, and educational level) and study endpoints (compliance). Finally, a cluster analysis (hierarchical, $K$-mean) will be performed. Cluster analysis is an exploratory data analysis tool that aims at sorting different objects (eg, traits, characteristics) into groups in a way that the degree of association between two objects is maximal if they belong to the same group and minimal otherwise. Given the above study objective, cluster analysis will permit identification of different compliance profiles, discovering the most peculiar traits and patients' characteristics in each cluster.

All statistical analysis will be performed using SAS/STAT software version 9.1 (SAS Institute Inc., Cary, NC, USA). The Proc GLIMMIX was used to fit mixed methods.

\section{Project activities Data handling procedures}

A documentation form will be completed for each patient. The documentation forms will be reviewed for accuracy and completeness by the Principal Investigator (PI). Computeraided data validations using preset procedures will be performed on an ongoing basis. Coding of medical terms and medication will be done using the current versions of coding dictionaries (ie, Medical Dictionary for Regulatory Activities, World Health Organization-Drug Dictionary enhanced). Questionnaire data will be transformed into SPSS for further and detailed analysis, which will be performed by the PI, who is expert in HRQL analysis.

\section{Ethical conduct and good clinical practice}

This study will be conducted in accordance with national laws. Ethics committees and national agencies will be informed or asked for approval. Regulations on data protection will be adhered to (eg, Directive 95/46/EC). This study is carried out completely independent of any medicinal treatment recommendations. Patients will be assessed and treated according to the individual decision of the attending physician. The study will not provide any recommendations with regard to diagnostic procedures or dosages, time of intake, or the strength of any medication.

\section{Data protection form}

It is the responsibility of the PI or a designated person (if acceptable according to local regulations), to obtain written 
Table 2 Gantt chart

\begin{tabular}{|c|c|c|c|c|c|c|c|c|c|c|c|c|c|}
\hline Task & Task name & M I & M 2 & M 3 & M 4 & M 5 & M 6 & M 7 & M 8 & M 9 & M IO & M II & M 12 \\
\hline I & Project management & & & & & & & & & & & & \\
\hline 2 & Protocol approval & & & & & & & & & & & & \\
\hline 3 & Patients' recruitment and assessment & & & & & & & & & & & & \\
\hline 3.1 & Patients' recruitment & & & & & & & & & & & & \\
\hline 3.2 & Test administration & & & & & & & & & & & & \\
\hline 4 & Data analysis and interpretation & & & & & & & & & & & & \\
\hline 4.1 & Data insertion and dataset & & & & & & & & & & & & \\
\hline 4.2 & Statistical analysis & & & & & & & & & & & & \\
\hline 5 & Exploitation and dissemination & & & & & & & & & & & & \\
\hline 5.1 & Conference and congress & & & & & & & & & & & & \\
\hline 5.2 & National seminars and meetings & & & & & & & & & & & & \\
\hline 5.3 & Paper preparation and submissions & & & & & & & & & & & & \\
\hline
\end{tabular}

Abbreviation: M, month.

consent to data collection from each patient participating in this study after adequate explanation of the objectives of data collection. It will also be explained to the patients that they will be fully free to allow or refuse the use of their data for the purposes of this study.

Appropriate forms for obtaining written consent to data collection will be provided to all patients.

\section{Timeline Duration}

The study period will be divided into two phases: a recruitment phase of up to 3 months, followed by a data collection phase of 6 months. The end of the data collection is defined as the last appointment of the last patient participating in the study (Table 2).

\section{Conclusion}

The project aims to evaluate patient compliance with treatment, which should help in the management of elderly patients with hemophilia. In Italy, treatment compliance in elderly hemophiliacs represents an innovative topic that is still understudied. No data are available and no information about risks and polytherapy are currently described in such a cohort. This study has the potential to evaluate compliance for the first time in Italian hemophilia centers and to be included in future studies conducted in the hemophilia community.

\section{Acknowledgments}

The first author acknowledges support from the World Federation of Hemophilia and from a Bayer Award program for young researchers, which together provided the opportunity to structure and conduct this study. The first author thanks Professor Mannuccio Mannucci for his important suggestions in implementing this paper.

\section{Disclosure}

The authors report no conflicts of interest in this work.

\section{References}

1. Mannucci PM, Schutgens RE, Santagostino E, Mauser-Bunschoten EP. How I treat age-related morbidities in elderly persons with hemophilia. Blood. 2009;114(26):5256-5263.

2. Tagliaferri A, Franchini M, von Mackensen S, et al. Health status and quality of life in elderly Italian patients with hemophilia. World Federation of Hemophilia World Congress; 2006 May 21-25; Vancouver, BC. Haemophilia. 2006;12(Suppl 2):252.

3. Baldi P, Iannello P, Riva S, Antonietti A. Cognitive reflection and socially biased decisions. Stud Psychol. 2013;55:265-271.

4. Riva S, Bullinger M, Amann E, von Mackensen S. Content comparison of haemophilia specific patient-rated outcome measures with the international classification of functioning, disability and health (ICF, ICF-CY). Health Qual Life Outcomes. 2010;8:139.

5. Mangiafico L, Perja M, Fusco F, Riva S, Mago D, Gringeri A. Safety and effectiveness of raltegravir in patients with haemophilia and antiHIV multidrug resistance. Haemophilia. 2012;18(1):108-111.

6. Krasuska M, Riva S, Fava L, von Mackensen S, Bullinger M. Linking quality-of-life measures using the International Classification of Functioning, Disability and Health and the International Classification of Functioning, Disability and Health-Children and Youth Version in chronic health conditions: the example of young people with hemophilia. Am J Phys Med Rehabil. 2012;91(13 Suppl 1):S74-S83.

7. Franchini M, Tagliaferri A, Mannucci PM. The management of hemophilia in elderly patients. Clin Interv Aging. 2007;2(3):361-368.

8. Muça-Perja M, Riva S, Grochowska B, Mangiafico L, Mago D, Gringeri A. Ultrasonography of haemophilic arthropathy. Haemophilia. 2012;18(3):364-368.

9. Kulkarni R, Soucie JM, Evatt BL; Hemophilia Surveillance System Project Investigators. Prevalence and risk factors for heart disease among males with hemophilia. Am J Hematol. 2005;79(1):36-42.

10. Riva S, Monti M, Iannello P, Antonietti A. The representation of risk in routine medical experience: what actions for contemporary health policy? PLoS One. 2012;7(11):e48297.

11. Riva S, Monti M, Iannello P, Pravettoni G, Schulz PJ, Antonietti A. A Preliminary Mixed-Method Investigation of Trust and Hidden Signals in Medical Consultations. PLoS ONE. 2014;9(3):e90941.

12. Chulz PJ, Hartung U, Riva S. Causes, Coping, and Culture: A Comparative Survey Study on Representation of Back Pain in Three Swiss Language Regions. PLoS ONE. 2013;8(11):e78029.

13. Riva S, Camerini AL, Allam A, Schulz PJ. Interactive Sections of an Internet-Based Intervention Increase Empowerment of Chronic Back Pain Patients: Randomized Controlled Trial. J Med Internet Res. 2014;16(8):e180 
14. Lindvall K, Colstrup L, Wollter IM, et al. Compliance with treatment and understanding of own disease in patients with severe and moderate haemophilia. Haemophilia. 2006;12(1):47-51.

15. Henriques MA, Costa MA, Cabrita J. Adherence and medication management by the elderly. J Clin Nurs. 2012;21(21-22):3096-3105.

16. Mackensen SV, Gringeri A. Quality of life in hemophilia. In: Preedy VR, Watson RR, editors. Handbook of Disease Burdens and Quality of Life Measures. New York, NY: Springer; 2010:1895-1920.

17. Risser J, Jacobson TA, Kripalani S. Development and psychometric evaluation of the Self-efficacy for Appropriate Medication Use Scale (SEAMS) in low-literacy patients with chronic disease. J Nurs Meas. 2007;15(3):203-219.

18. Mannucci PM, Nobili A; REPOSI Investigators. Multimorbidity and polypharmacy in the elderly: lessons from REPOSI. Intern Emerg Med. 2014;9(7):723-734.

19. Folstein MF, Folstein SE, McHugh PR. "Mini-mental state". A practical method for grading the cognitive state of patients for the clinician. J Psychiatr Res. 1975;12(3):189-198.
20. Devlin NJ, Krabbe PF. The development of new research methods for the valuation of EQ-5D-5L. Eur J Health Econ. 2013;14 Suppl 1: $\mathrm{S} 1-\mathrm{S} 3$.

21. Ozelo M, Regnault A, Slothuus U, Hoxer CS. Satisfaction of young adults with haemophilia A previously treated with prophylaxis and switching to prophylactic treatment with recombinant factor VIII turoctocog alfa. European Association for Haemophilia and Allied Disorders 8th Annual Congress; 2015 Feb 11-13; Helsinki, Finland. Haemophilia. 2015;21(Suppl S2):66-67. Abstract \#PP089.

22. Schrijvers LH, Uitslager N, Schuurmans MJ, Fischer K. Barriers and motivators of adherence to prophylactic treatment in haemophilia: a systematic review. Haemophilia. 2013;19(3):355-361.

23. Van Voorhis CRW, Morgan BL. Understanding power and rules of thumb for determining sample sizes. Tutor Quant Methods Psychol. 2007;3(2):43-50.
Clinical Interventions in Aging

\section{Publish your work in this journal}

Clinical Interventions in Aging is an international, peer-reviewed journal focusing on evidence-based reports on the value or lack thereof of treatments intended to prevent or delay the onset of maladaptive correlates of aging in human beings. This journal is indexed on PubMed Central, MedLine,

\section{Dovepress}

CAS, Scopus and the Elsevier Bibliographic databases. The manuscript management system is completely online and includes a very quick and fair peer-review system, which is all easy to use. Visit http://www.dovepress. com/testimonials.php to read real quotes from published authors. 